\title{
Photon shuttle: Landau-Zener-Stückelberg dynamics in an optomechanical system
}

\author{
Georg Heinrich, ${ }^{1}$ J. G. E. Harris, ${ }^{2,3}$ and Florian Marquardt ${ }^{1}$ \\ ${ }^{1}$ Arnold Sommerfeld Center for Theoretical Physics, Center for NanoScience and Department of Physics, \\ Ludwig-Maximilians-Universität München, Theresienstr. 37, D-80333 München, Germany \\ ${ }^{2}$ Department of Physics, Yale University, 217 Prospect Street, New Haven, Connecticut 06520, USA \\ ${ }^{3}$ Department of Applied Physics, Yale University, 15 Prospect Street, New Haven, Connecticut 06520, USA
}

(Received 16 September 2009; published 5 January 2010)

\begin{abstract}
The motion of micro- and nanomechanical resonators can be coupled to electromagnetic fields. Such optomechanical setups allow one to explore the interaction of light and matter in a new regime at the boundary between quantum and classical physics. We propose an approach to investigate nonequilibrium photon dynamics driven by mechanical motion in a recently developed setup with a membrane between two mirrors, where photons can be shuttled between the two halves of the cavity. For modest driving strength we predict the possibility of observing an Autler-Townes splitting indicative of Rabi dynamics. For large drive, we show that this system displays Landau-Zener-Stueckelberg dynamics originally known from atomic two-state systems.
\end{abstract}

DOI: 10.1103/PhysRevA.81.011801

PACS number(s): 42.50.Hz, 07.10.Cm, 42.65.Sf

Optomechanical systems couple mechanical degrees of freedom to radiation fields and constitute a rapidly evolving field of current research (reviewed in [1]). These systems provide new means to manipulate both the light field and the mechanical motion. Apart from the hope of eventually exploring the quantum regime of mechanical motion, there have been several studies of the complex nonlinear dynamics of these systems [2-6]. An exciting recent development is the introduction of setups with multiple coupled optical (and vibrational) modes, pointing the way toward integrated optomechanical circuits [7-10]. In this article, we show how the application of an external mechanical drive to these structures can open up the whole domain of strongly driven two-level and multilevel systems to the field of optomechanics. As a concrete example of such a mechanically driven coherent photon dynamics, we consider the system recently realized in $[7,11]$, where we show that a vibrating membrane inside an optical cavity can shuttle photons between two optical modes. We predict Autler-Townes splittings due to Rabi processes and Landau-Zener-Stueckelberg (LZS) oscillations visible in the transmission spectrum. Landau-Zener (LZ) transitions [12,13] and LZS oscillations [14] were originally studied in atomic systems [15-17], but lately they have also been applied to quantum dots and superconducting qubits [18-21]. Some purely optical setups [22,23] have also mimicked two-level and standard LZ dynamics, but not LZS oscillations. More generally, the mechanically driven coherent photon dynamics in multimode optomechanical systems introduced in this article will allow one to realize analogs to driven atomic multilevel systems, such as $V$-type and $\Lambda$-type level schemes and effects such as coherent trapping or electromagnetically induced transparency.

We consider two cavity modes coupled by a dielectric membrane placed between two high-finesse mirrors [see Fig. 1(a)]. The system Hamiltonian is

$$
\begin{aligned}
\hat{H}_{\text {sys }}= & \hbar \omega_{0}\left[1-\frac{x(t)}{l}\right] \hat{a}_{L}^{\dagger} \hat{a}_{L}+\hbar \omega_{0}\left[1+\frac{x(t)}{l}\right] \hat{a}_{R}^{\dagger} \hat{a}_{R} \\
& +\hbar g\left(\hat{a}_{L}^{\dagger} \hat{a}_{R}+\hat{a}_{R}^{\dagger} \hat{a}_{L}\right)+\hat{H}_{\text {drive }}+\hat{H}_{\text {decay }} .
\end{aligned}
$$

$\hat{a}_{L}^{\dagger} \hat{a}_{L}$ and $\hat{a}_{R}^{\dagger} \hat{a}_{R}$ are the photon numbers for the two optical modes in the left and right cavity half (each of length $l$ ), respectively, whose resonance frequency $\omega_{0}$ is changed due to the displacement $x$ of the membrane. The coupling $g$ describes photon tunneling through the membrane. Due to the coupling, there is an avoided crossing in the optical resonance frequency $\omega_{ \pm}(x)= \pm \sqrt{g^{2}+\left(\omega_{0} x / l\right)^{2}}$ [see Fig. 1(b)]. We propose to drive the membrane with mechanical frequency $\Omega$ and resulting amplitude $A$ around a mean position $x_{0}$,

$$
x(t)=A \cos (\Omega t)+x_{0},
$$

and investigate photon dynamics in terms of the cavity transmission in the regime where the time scale of photon exchange is comparable to the time scale of the mechanical motion $(g \simeq \Omega$ ). Recently, the coupling frequency $g / 2 \pi$ has been significantly reduced by exploiting properties of transverse modes [23], and it is tunable down to $200 \mathrm{kHz}$ at present. The mechanical eigenfrequencies of typical $1 \mathrm{~mm} \times 1 \mathrm{~mm} \times 50 \mathrm{~nm}$

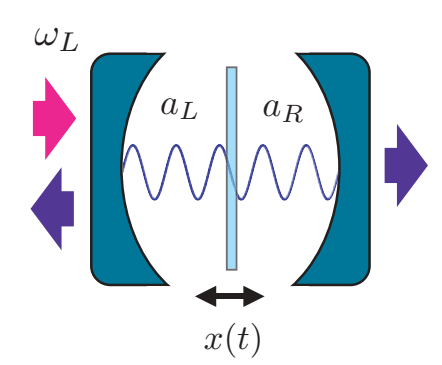

(a)

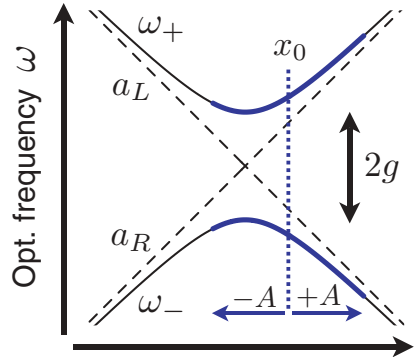

(b)

Displacement $x$
FIG. 1. (Color online) (a) Setup. A dielectric membrane couples two modes $a_{L}, a_{R}$ inside a cavity. The left-hand side is excited by a laser $\omega_{L}$ while the transmission to the right is recorded. (b) Optical resonance frequency as function of displacement. The membrane's displacement linearly changes the bare mode frequencies (dashed). Due to the coupling $g$, there is an avoided crossing of the eigenfrequencies $\omega_{ \pm}$(black). The membrane is driven, with $x(t)=A \cos (\Omega t)+x_{0}$ (blue; thicker). 
membranes range between $100 \mathrm{kHz}$ and $1 \mathrm{MHz}$. We point out that here $\Omega$ need not coincide with the membrane's eigenfrequency but depends only on the driving. In the experimental scheme, we propose to optically drive the left-hand side of the cavity with a laser of tunable frequency $\omega_{L}$ and measure the transmission $T$ to the right with a photo detector placed on the other side of the cavity [see Fig. 1(a)]. The mechanical driving Eq. (2) might be realized by mounting the membrane on a piezo actuator.

Using input-output theory, the equations of motion for the average fields $a_{L}=\left\langle\hat{a}_{L}\right\rangle$ and $a_{R}=\left\langle\hat{a}_{R}\right\rangle$ read

$$
\begin{gathered}
\frac{d}{d t} a_{L}=\frac{1}{i}\left[-\bar{x}(t) a_{L}+g a_{R}\right]-\frac{\kappa}{2} a_{L}-\sqrt{\kappa} b_{L}^{i n}(t) \\
\frac{d}{d t} a_{R}=\frac{1}{i}\left[+\bar{x}(t) a_{R}+g a_{L}\right]-\frac{\kappa}{2} a_{R},
\end{gathered}
$$

with the cavity decay rate $\kappa$ for each of the modes, and laser drive $b_{L}^{i n}(t)=e^{-i \Delta_{L} t} b^{i n}$ with amplitude $b^{i n}$. Here, we used a rotating frame, with laser detuning from resonance $\Delta_{L}=\omega_{L}-\omega_{0}$. The displacement is written in terms of a frequency via $\bar{x}(t)=\left(\omega_{0} / l\right) x(t)$; likewise for $\bar{A}, \bar{x}_{0}$. The transmission to the right, $T(t)=\kappa\left\langle\hat{a}_{R}^{\dagger}(t) \hat{a}_{R}(t)\right\rangle /\left(b^{i n}\right)^{2}$, can be expressed as

$$
T(t)=\kappa^{2}\left|\int_{-\infty}^{t} G\left(t, t^{\prime}\right) e^{-i \Delta_{L} t^{\prime}-(\kappa / 2)\left(t-t^{\prime}\right)} d t^{\prime}\right|^{2},
$$

where the phase factor includes laser drive and cavity decay, while the Green's function $G\left(t, t^{\prime}\right)$ describes the amplitude for a photon to enter the cavity from the left at time $t^{\prime}$ and to be found in the right cavity mode later at time $t$. Technically, $G\left(t, t^{\prime}\right)$ is found by setting $\kappa=0$ in Eq. (3) and solving for $a_{R}(t)$ with the initial conditions $a_{L}\left(t^{\prime}\right)=1, a_{R}\left(t^{\prime}\right)=0$.

Figure 2(a) displays the time-averaged transmission depending on $\bar{x}_{0}$ and $\Delta_{L}$ without mechanical drive $(\bar{A}=0)$. The spectrum displays the two hyperbola branches $\omega_{ \pm}$[Fig. 1(b)]. Transmission is largest at the avoided crossing where photons can most easily tunnel from the left into the right mode. For modest drive $\bar{A}<\Omega$, Fig. 2(b) shows an Autler-Townes splitting $[22,25]$ of the two hyperbola branches $\omega_{ \pm}$. Indeed, if resonant, the mechanical drive induces Rabi oscillations between the two photon branches, at a Rabi frequency $g_{1} \simeq$ $g \bar{A} / \Omega$ [see Eq. (9)], leading to a corresponding splitting in the spectroscopic picture. Furthermore, the mechanically assisted process enables high transmission even farther away from the anti-crossing. For larger drive amplitudes [see Fig. 2(c)], the dynamics becomes more involved as mechanical sidebands arise and interact with each other. In the following, we will focus on the dynamics of the strong driving regime.

Figure 3 shows numerical results for $\bar{A} \gg \Omega, g$ and experimentally accessible parameters (for $g / 2 \pi=1 \mathrm{MHz}$, $l=1 \mathrm{~cm}, \omega_{0} / 2 \pi=3 \times 10^{14} \mathrm{~Hz} ; \bar{A}=60 \mathrm{~g}$ corresponds to an oscillation amplitude $A=2 \mathrm{~nm}$ ). We first give an intuitive description of why finite transmission $T$ can be observed only if $\bar{x}_{0}$ is a multiple of $\Omega$, and we comment later on the modulation as a function of $\bar{A}$. T is determined by two subsequent processes: first, the laser has to excite the left mode; second, the internal dynamics must be able to transfer photons into the right one. In general, both processes

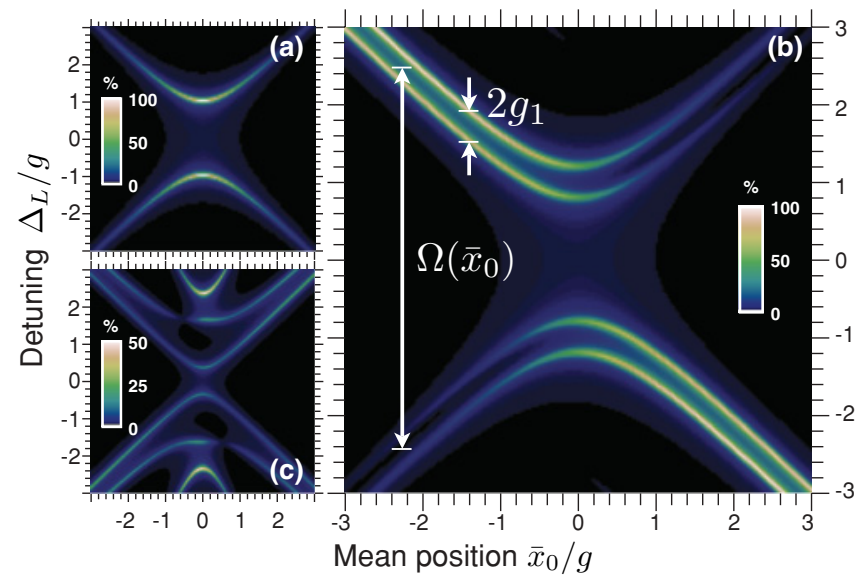

FIG. 2. (Color online) Density plot for the time-averaged transmission depending on mean position $\bar{x}_{0}$ and laser detuning $\Delta_{L}$ for cavity decay rate $\kappa=0.1 \mathrm{~g}$. (a) Without mechanical drive $\bar{A}=0$, the spectrum is given by $\omega_{ \pm}$[see Fig. 1(b)]. (b) Autler-Townes splitting of the cavity frequency $\omega_{ \pm}$due to mechanical motion, $\bar{A}=0.2 \Omega$. For every position $\bar{x}_{0}$ the mechanical drive frequency is set to be $\Omega=2 \sqrt{g^{2}+\bar{x}_{0}^{2}}$ such that it is always resonant with the difference between the two optical mode frequencies $\omega_{ \pm}$. The splitting is set by the Rabi frequency $g_{1} \simeq g \bar{A} / \Omega$ [see Eq. (9)]. (c) Plot as in panel (b) but for stronger drive $\bar{A}=1.6 \Omega$. Mechanical sidebands, displaced by $\pm \Omega$, become visible and interact.

are inelastic and therefore require energy to be exchanged between the light field and the mechanics. The left mode's frequency is oscillating around the time-averaged value $-\bar{x}_{0}$. Hence, the resonance condition to excite the left mode reads

$$
\Delta_{L}+m \Omega=-\bar{x}_{0},
$$

[see Fig. 4(a)]. Here, $m \Omega$ is an adequate multiphonon transition. The width of the individual resonances is determined by $\kappa$. The subsequent process displays the physics of LZS dynamics: in general, if a parameter in a two-state system is swept sufficiently fast through an avoided crossing, the system may undergo an LZ transition into the other eigenstate [12,13]. Here, for periodic sweep, we face iterated transitions. The first LZ transition splits the photon state into a coherent superposition; the two amplitudes gather different phases and interfere the next time the system transverses the avoided crossing. For two-state systems the resulting interference patterns in the state population are known as Stueckelberg oscillations [14]. The condition for constructive interference can also be phrased in terms of an additional multiphonon transition that transfers a photon from the left mode with average frequency $-\bar{x}_{0}$ to the right one at $+\bar{x}_{0}$,

$$
n \Omega=2 \bar{x}_{0} .
$$

We find transmission only if both conditions are met. We note that the coupling $g$ between modes does not enter here. We will come back to this point later.

To derive these resonance conditions as well as to understand the dependence on $\bar{A}$, in the following, we calculate an approximate, analytic expression for the transmission. 


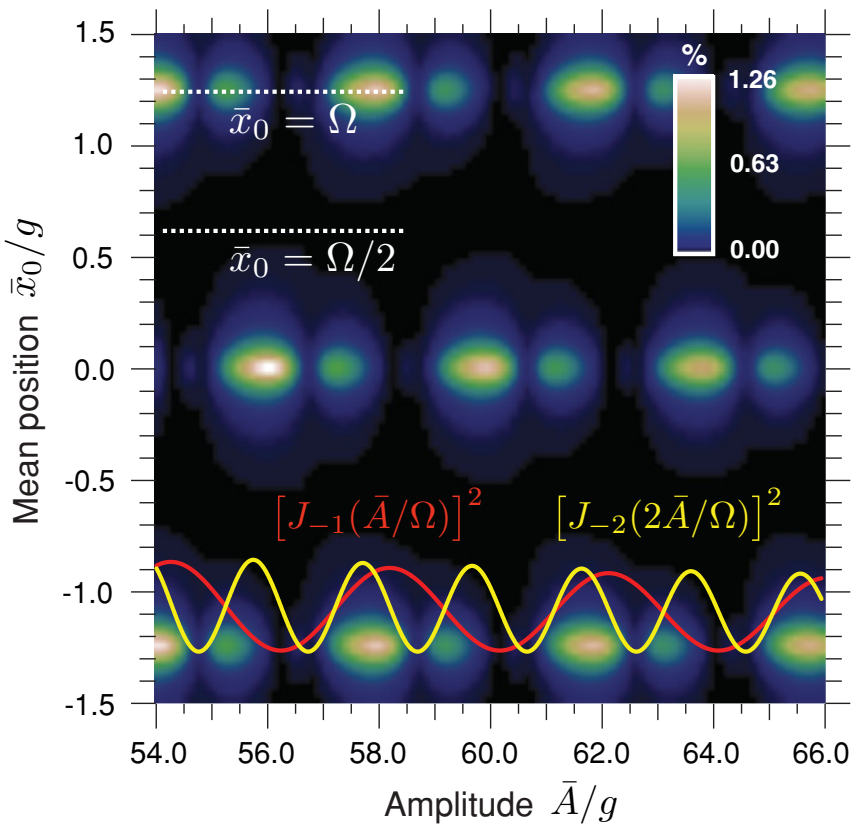

FIG. 3. (Color online) Density plot for the time-averaged transmission as function of average displacement $\bar{x}_{0}$ and mechanical drive amplitude $\bar{A} \gg \Omega, g$. Further parameters are laser detuning $\Delta_{L}=0$, mechanical frequency $\Omega / 2 \pi=0.2 g$, and cavity decay $\kappa=0.2 g$. Finite transmission is observed when the resonance conditions Eqs. (5) and (6) for multiphonon transitions are met. The transmission is modulated according to the product of two Bessel functions. For the case $\bar{x}_{0}=-\Omega$, both are depicted in the plot's plane. Red (longer period): $\sim J_{m}^{2}(\bar{A} / \Omega)$, due to the excitation process. Yellow (shorter period): $\sim J_{n}^{2}(2 \bar{A} / \Omega)$, due to LZS dynamics.

From Eq. (3), the Green's function $G\left(t, t^{\prime}\right)$, required for the transmission (4), is found to be

$$
G\left(t, t^{\prime}\right)=\tilde{a}_{R}\left(t, t^{\prime}\right) e^{-i \phi\left(t^{\prime}\right)},
$$

where we have split off a phase $\phi\left(t^{\prime}\right)=(\bar{A} / \Omega) \sin \left(\Omega t^{\prime}\right)$, and $\tilde{a}_{R}\left(t, t^{\prime}\right)$ is a solution to

$$
i \frac{d}{d t}\left(\begin{array}{c}
\tilde{a}_{R} \\
\tilde{a}_{L}
\end{array}\right)=\left(\begin{array}{cc}
\bar{x}_{0} & g e^{+2 i \phi(t)} \\
g e^{-2 i \phi(t)} & -\bar{x}_{0}
\end{array}\right)\left(\begin{array}{c}
\tilde{a}_{R} \\
\tilde{a}_{L}
\end{array}\right),
$$

with $t \geqslant t^{\prime}$ and initial condition $\tilde{a}_{R}\left(t^{\prime}, t^{\prime}\right)=0, \tilde{a}_{L}\left(t^{\prime}, t^{\prime}\right)=1$. We now show that the two multiphonon processes introduced above correspond to the two factors in Eq. (7). The term $e^{-i \phi\left(t^{\prime}\right)}=\sum_{m} J_{m}(\bar{A} / \Omega) e^{-i m \Omega t^{\prime}}$ describes the initial excitation, where the amplitude for a transfer of $m$ phonons is set by the Bessel function $J_{m}(\bar{A} / \Omega)$. Second, the internal dynamics described by $\tilde{a}_{R}\left(t, t^{\prime}\right)$ is expressed in terms of a two-level system with time-dependent coupling $g e^{2 i \phi(t)}=$ $g \sum_{n} J_{n}(2 \bar{A} / \Omega) e^{i n \Omega t}$. Thus, the strength of the second multiphonon transition $n \Omega$ in Fig. 4(a) is determined by a Bessel function $J_{n}(2 \bar{A} / \Omega)$. As a special case, this also describes the Autler-Townes splitting at small drive. This can be calculated from (8) using an interaction picture representation and considering the time-dependent coupling only up to $J_{1}$, yielding an effective transition frequency $2 \sqrt{g_{0}^{2}+\bar{x}_{0}^{2}}$, with $g_{0}=g J_{0}(2 \bar{A} / \Omega)$, and a Rabi frequency $g_{1}=g J_{1}(2 \bar{A} / \Omega)$.
In the case of LZS dynamics (i.e., strong drive), for sufficiently large amplitudes only one of the harmonics of $g \sum_{n} J_{n}(2 \bar{A} / \Omega) e^{i n \Omega t}$ will be in resonance with the system. This corresponds to leading-order perturbation theory within the Floquet approach [26] applied to Eq. (8). In this case, Eq. (8) simplifies to the problem of a two-state system with harmonic drive at $n \Omega$ and effective coupling constant

$$
g_{n}=g J_{n}(2 \bar{A} / \Omega) .
$$

To estimate when this approximation becomes appropriate, we note that for a driven undamped two-state system the width of the power-broadened resonance is set by the Rabi frequency. Thus, Eq. (8) yields a series of resonance peaks at $\bar{x}_{0}=n \Omega / 2$, and they become separated if $4 g_{n}<\Omega$. Using the asymptotic form for large arguments $\bar{A} / \Omega \gg 1, J_{n}(y) \simeq$ $\sqrt{\frac{2}{\pi y}} \cos \left(y-\frac{n \pi}{2}-\frac{\pi}{4}\right)$, we find the resonance approximation to hold whenever $g^{2}<(\pi / 16) \bar{A} \Omega$. This is clearly fulfilled for the parameters in Fig. 3. Note the resemblance to the criterion for nonadiabatic transitions that can be derived from the standard LZ formula $P_{1 \rightarrow 1}=\exp \left(-\pi g^{2} / 2 v\right)$, where $v=\bar{A} \Omega$ is the sweep velocity.

Given the resonance approximation, we find for the Green's function

$$
G\left(t, t^{\prime}\right)=-i \frac{g_{n}}{\omega_{n}} \sin \left[\omega_{n}\left(t-t^{\prime}\right)\right] e^{-i n \Omega\left(t+t^{\prime}\right) / 2} e^{-i \phi\left(t^{\prime}\right)},
$$

with $\omega_{n}=\sqrt{\left(g_{n}\right)^{2}+\left(\bar{x}_{0}-n \Omega / 2\right)^{2}}$. Note that $\omega_{n}$ contains $g_{n}$, which is much smaller than the bare splitting $g$ for $\bar{A} \gg \Omega$. This explains why the resonance conditions (5) and (6) involve the bare optical mode frequencies $\pm \bar{x}_{0}$ instead of the adiabatic eigenfrequencies $\omega_{ \pm}$.

We insert (10) into (4), taking into account the sum over independent contributions with $n$ quanta. In the resolved sideband regime $(\Omega>\kappa)$, the integration of (4) selects a specific $m$ for the excitation process [see Eq. (5)]. We find an approximate expression (displayed here for the special case $\Delta_{L}=0$, where $n=2 m$ ),

$$
\begin{aligned}
T= & \left(\frac{\kappa}{g}\right)^{2} \sum_{m}\left\{J_{m}\left(\frac{\bar{A}}{\Omega}\right)\right. \\
& \left.\times \frac{J_{2 m}\left(2 \frac{\bar{A}}{\Omega}\right)}{\frac{1}{g^{2}}\left[\left(\frac{\kappa}{2}\right)^{2}+\left(x_{0}-m \Omega\right)^{2}\right]+\left[J_{2 m}\left(2 \frac{\bar{A}}{\Omega}\right)\right]^{2}}\right\}^{2},
\end{aligned}
$$

that fully captures the numerical results shown in Fig. 3. In contrast to $J_{m}(\bar{A} / \Omega)$, the LZS dynamics, characterized by $J_{n}(2 \bar{A} / \Omega)$, involves $2 \bar{A}$ as it is determined by the phase difference gathered between $L Z$ transitions. If we were to increase $\Delta_{L}$ in Fig. 3, we would tune out of resonance and the transmission would vanish everywhere. For $\Delta_{L}=\Omega / 2$, (5) and (6) can be met for $\bar{x}_{0}$ being an odd multiple of $\Omega / 2$. For $\Delta_{L}=\Omega$, we recover the resonances of Fig. 3; however, the entire plot would be shifted in $\bar{A}$ by $\pi \Omega / 2$ due to the changed index of the Bessel function $J_{m}$. The qualitative result at $\Delta_{L}=2 \Omega$ finally resembles the one shown in Fig. 3 . Finally, Fig. 4(b) shows numerical results for smaller values 


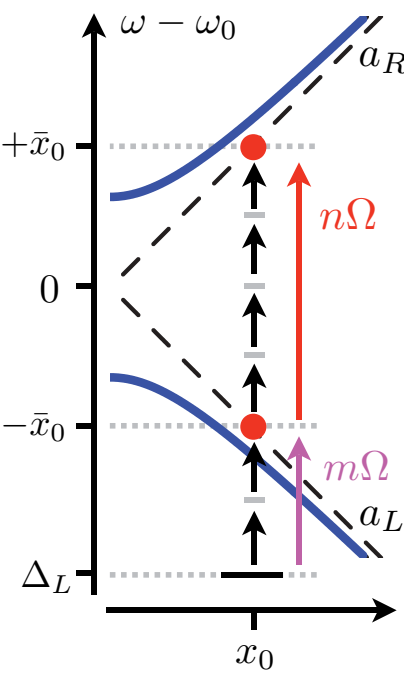

(a)

Displacement

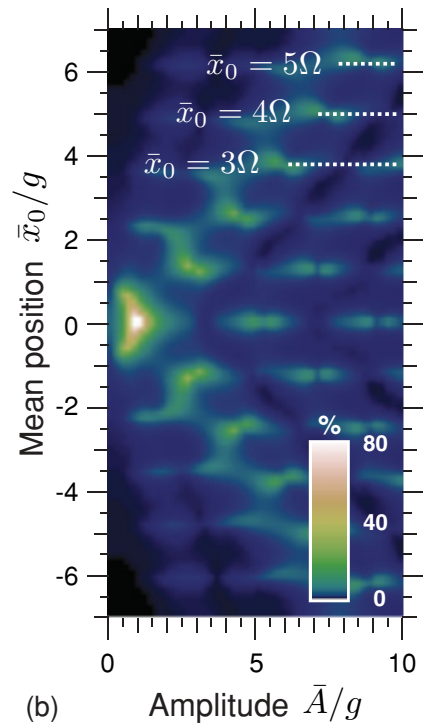

FIG. 4. (Color online) (a) Multiphonon transition picture. To see transmission, two processes are involved: First (magenta, labeled $m \Omega$ ), excitation of the left cavity mode by the laser drive at $\Delta_{L}$, supported by $m$ phonons. Second (red, labeled $n \Omega$ ), a suitable $n$-quanta multiphonon transition to transfer a photon from the left into the right mode. (b) Density plot for time-averaged transmission as a function of $\bar{x}_{0}$ and $\bar{A} \simeq g$. Further parameters as in Fig. 3 . of $\bar{A} / g$ (while keeping $\Omega$ as in Fig. 3). As before, we see resonances of width $\kappa$ for $\bar{x}_{0}$ being a multiple of $\Omega$ and regions of excitation determined by $J_{m}(\bar{A} / \Omega)$. Within these regions, we note the already familiar substructure that is due to LZS dynamics.

To conclude, we have introduced mechanically driven coherent photon dynamics for multimode optomechanical systems. For a specific setup we predicted Autler-Townes splittings and features of LZS dynamics whose observation is within reach of current experiments. We note that the same photon dynamics will enter when describing self-induced nonlinear optomechanical oscillations in these systems. Future optomechanical circuits [8-10] that integrate multiple optical and vibrational modes on a chip will thus allow one to transfer the whole realm of strongly driven multilevel dynamics from atomic physics into the world of optomechanics. The tunability and custom design of optomechanical systems, as well as their ability to couple to each other, will offer a new regime in which to explore these phenomena.

We acknowledge fruitful discussions with Jack Sankey, as well as support by the DFG (SFB 631, SFB TR 12, NIM, Emmy-Noether program) and DIP and (J.H.) via NSF 0855455, NSF 0653377, and AFOSR FA9550-09-10484.
[1] F. Marquardt and S. M. Girvin, Physics 2, 40 (2009).

[2] T. Carmon, H. Rokhsari, L. Yang, T. J. Kippenberg, and K. J. Vahala, Phys. Rev. Lett. 94, 223902 (2005).

[3] T. J. Kippenberg, H. Rokhsari, T. Carmon, A. Scherer, and K. J. Vahala, Phys. Rev. Lett. 95, 033901 (2005).

[4] F. Marquardt, J. G. E. Harris, and S. M. Girvin, Phys. Rev. Lett. 96, 103901 (2006).

[5] C. Metzger, M. Ludwig, C. Neuenhahn, A. Ortlieb, I. Favero, K. Karrai, and F. Marquardt, Phys. Rev. Lett. 101, 133903 (2008).

[6] M. Ludwig, B. Kubala, and F. Marquardt, New J. Phys. 10, 095013 (2008).

[7] J. D. Thompson, B. M. Zwickl, A. M. Jayich, F. Marquardt, S. M. Girvin, and J. G. E. Harris, Nature (London) 452, 72 (2008).

[8] M. Li, W. H. P. Pernice, C. Xiong, T. Baehr-Jones, M. Hochberg, and H. X. Tang, Nature (London) 456, 480 (2008).

[9] M. Eichenfield, R. Camacho, J. Chan, K. J. Vahala, and O. Painter, Nature (London) 459, 550 (2009).

[10] M. Eichenfield, J. Chan, R. M. Camacho, K. J. Vahala, and O. Painter, Nature (London) 462, 78 (2009).

[11] A. M. Jayich, J. C. Sankey, B. M. Zwickl, C. Yang, J. D. Thompson, S. M. Girvin, A. A. Clerk, F. Marquardt, and J. G. E. Harris, New J. Phys. 10, 095008 (2008).

[12] L. D. Landau, Phys. Z. Sowjetunion 2, 46 (1932).

[13] C. Zener, Proc. R. Soc. London A 137, 696 (1932).

[14] E. C. G. Stückelberg, Helv. Phys. Acta 5, 369 (1932).
[15] M. C. Baruch and T. F. Gallagher, Phys. Rev. Lett. 68, 3515 (1992).

[16] S. Yoakum, L. Sirko, and P. M. Koch, Phys. Rev. Lett. 69, 1919 (1992).

[17] M. Mark, T. Kraemer, P. Waldburger, J. Herbig, C. Chin, H.-C. Nägerl, and R. Grimm, Phys. Rev. Lett. 99, 113201 (2007).

[18] W. D. Oliver, Y. Yu, J. C. Lee, K. K. Berggren, L. S. Levitov, and T. P. Orlando, Science 310, 1653 (2005).

[19] K. Saito, M. Wubs, S. Kohler, P. Hänggi, and Y. Kayanuma, Europhys. Lett. 76, 22 (2006).

[20] M. Wubs, K. Saito, S. Kohler, P. Hanggi, and Y. Kayanuma, Phys. Rev. Lett. 97, 200404 (2006).

[21] D. M. Berns, M. S. Rudner, S. O. Valenzuela, K. K. Berggren, W. D. Oliver, L. S. Levitov, and T. P. Orlando, Nature (London) 455, 51 (2008).

[22] R. J. C. Spreeuw, N. J. vanDruten, M. W. Beijersbergen, E. R. Eliel, and J. P. Woerdman, Phys. Rev. Lett. 65, 2642 (1990).

[23] D. Bouwmeester, N. H. Dekker, F. E. v. Dorsselaer, C. A. Schrama, P. M. Visser, and J. P. Woerdman, Phys. Rev. A 51, 646 (1995).

[24] J. C. Sankey, A. M. Jayich, B. M. Zwickl, C. Yang, and J. G. E. Harris, in Proceedings of the XXI International Conference on Atomic Physics, edited by R. Cote, P. L. Gould, M. Rozman, and W. W. Smith (World Scientific, Singapore, 2009).

[25] S. H. Autler and C. H. Townes, Phys. Rev. 100, 703 (1955).

[26] M. Grifoni and P. Hänggi, Phys. Rep. 304, 229 (1998). 